\title{
The association of neutrophil to lymphocyte ratio, platelet to lymphocyte ratio, and lymphocyte to monocyte ratio with post-thrombolysis early neurological outcomes in patients with acute ischemic stroke
}

Pengyu Gong ${ }^{1 \dagger}$, Yukai Liu ${ }^{1 \dagger}$, Yachi Gong ${ }^{2}$, Gang Chen ${ }^{3}$, Xiaohao Zhang ${ }^{4}$, Siyu Wang ${ }^{1}$, Feng Zhou', Rui Duan ${ }^{5}$, Wenxiu Chen ${ }^{6}$, Ting Huang ${ }^{1}$, Meng Wang ${ }^{1}$, Qiwen Deng ${ }^{1}$, Hongchao Shi ${ }^{1}$, Junshan Zhou ${ }^{1 *}$, Teng Jiang ${ }^{1 *}$ and Yingdong Zhang ${ }^{1 *}$

\begin{abstract}
Background and purpose: To investigate the association of neutrophil to lymphocyte ratio (NLR), platelet to lymphocyte ratio (PLR), and lymphocyte to monocyte ratio (LMR) with post-thrombolysis early neurological outcomes including early neurological improvement (ENI) and early neurological deterioration (END) in patients with acute ischemic stroke (AIS).

Methods: AIS patients undergoing intravenous thrombolysis were enrolled from April 2016 to September 2019. Blood cell counts were sampled before thrombolysis. Post-thrombolysis END was defined as the National Institutes of Health Stroke Scale (NIHSS) score increase of $\geq 4$ within $24 \mathrm{~h}$ after thrombolysis. Post-thrombolysis ENI was defined as NIHSS score decrease of $\geq 4$ or complete recovery within $24 \mathrm{~h}$. Multinomial logistic regression analysis was performed to explore the relationship of NLR, PLR, and LMR to post-thrombolysis END and ENI. We also used receiver operating characteristic curve analysis to assess the discriminative ability of three ratios in predicting END and ENI.
\end{abstract}

(Continued on next page)

\footnotetext{
* Correspondence: zhoujunshan6807@163.com; jiang_teng@njmu.edu.cn; zhangyingdong@aliyun.com

${ }^{\dagger}$ Pengyu Gong and Yukai Liu contributed equally to this work.

'Department of Neurology, Nanjing First Hospital, Nanjing Medical University,

Nanjing 210006, Jiangsu, China

Full list of author information is available at the end of the article
}

(c) The Author(s). 2021 Open Access This article is licensed under a Creative Commons Attribution 4.0 International License, which permits use, sharing, adaptation, distribution and reproduction in any medium or format, as long as you give appropriate credit to the original author(s) and the source, provide a link to the Creative Commons licence, and indicate if changes were made. The images or other third party material in this article are included in the article's Creative Commons licence, unless indicated otherwise in a credit line to the material. If material is not included in the article's Creative Commons licence and your intended use is not permitted by statutory regulation or exceeds the permitted use, you will need to obtain permission directly from the copyright holder. To view a copy of this licence, visit http://creativecommons.org/licenses/by/4.0/. The Creative Commons Public Domain Dedication waiver (http://creativecommons.org/publicdomain/zero/1.0/) applies to the data made available in this article, unless otherwise stated in a credit line to the data. 
(Continued from previous page)

Results: Among 1060 recruited patients, a total of 193 (18.2\%) were diagnosed with END and 398 (37.5\%) were diagnosed with ENI. Multinomial logistic model indicated that NLR (odds ratio [OR], 1.385; 95\% confidence interval [Cl] 1.238-1.551, $P=0.001), \operatorname{PLR}(\mathrm{OR}, 1.013 ; 95 \% \mathrm{Cl} 1.009-1.016, P=0.001)$, and LMR (OR, 0.680; 95\% Cl 0.560-0.825, $P=0.001)$ were independent factors for post-thrombolysis END. Moreover, NLR (OR, 0.713; 95\% Cl 0.643-0.791, $P=$ 0.001) served as an independent factor for post-thrombolysis ENI. Area under curve (AUC) of NLR, PLR, and LMR to discriminate END were $0.763,0.703$, and 0.551 , respectively. AUC of NLR, PLR, and LMR to discriminate ENI were $0.695,0.530$, and 0.547 , respectively.

Conclusions: NLR, PLR, and LMR were associated with post-thrombolysis END. NLR and PLR may predict postthrombolysis END. NLR was related to post-thrombolysis ENI.

Keywords: Acute ischemic stroke, Early neurological deterioration, Early neurological improvement, Intravenous thrombolysis, Neutrophil to lymphocyte ratio, Platelet to lymphocyte ratio, Lymphocyte to monocyte ratio

\section{Introduction}

Stroke is one of the main reasons for mortality and morbidity at the national level in China [1, 2]. The efficacy of thrombolysis with intravenous recombinant tissue plasminogen activator was demonstrated for the patients with acute ischemic stroke (AIS) [3-5]. It has been reported that rapid recovery, which is described as early neurological improvement (ENI), can be observed in a significant proportion of AIS patients within the first 24 $\mathrm{h}$ after intravenous thrombolysis [3, 4]. However, there are still several patients whose symptoms would worsen and neurological deficits may aggravate within $24 \mathrm{~h}$ after intravenous thrombolysis, named as early neurological deterioration (END) [6-8]. Previous researches indicate that post-thrombolysis early neurological outcomes, including post-thrombolysis END as well as postthrombolysis ENI, are relevant to the prognosis of patients treated with intravenous thrombolysis [9, 10]. Post-thrombolysis ENI can promote acceptable longterm outcomes for the patients with AIS [9], and END results in the increasing likelihood of mortality and morbidity [10]. Therefore, it is significant to explore the risk factors and the measurable biomarkers of postthrombolysis early neurological outcomes in AIS patients.

Numerous studies have demonstrated that neuroinflammatory response plays an essential role in the pathophysiology of ischemic stroke [11-14]. Neutrophil to lymphocyte ratio (NLR), platelet to lymphocyte ratio (PLR), and lymphocyte to monocyte ratio (LMR) have recently been reported as potential novel biomarkers of baseline inflammatory process and could serve as outstanding predictors in patients with ischemic stroke [15, 16]. NLR, PLR, and LMR may have the ability to predict functional outcome in AIS patients treated with intravenous thrombolysis [17-19]. Goyal and colleagues found NLR on admission may be able to function as a prognostic biomarker of outcomes in patients with large vessel occlusion stroke [20]. What is more, previous studies showed these three novel composite inflammatory ratios, which were mentioned above, could have superior predictive capacity to those of traditional inflammatory factors [21]. Nevertheless, the relationship between these composite inflammatory ratios and postthrombolysis early neurological outcomes in AIS patients remains uncertain and worth exploring.

In this study, we aimed to investigate the association of composite inflammatory ratios before thrombolysis, including NLR, PLR, and LMR, with post-thrombolysis END as well as ENI in AIS patients. Furthermore, we planned to explore the utility of these composite inflammatory ratios in predicting post-thrombolysis END and post-thrombolysis ENI.

\section{Methods \\ Study design and participants}

AIS patients undergoing intravenous thrombolysis within $4.5 \mathrm{~h}$ were recruited from the Nanjing First Hospital, Haimen Hospital Affiliated to Nantong University and Nantong Third Peoples Hospital. All the patients were treated in the stroke units and received standard treatments, for instance, antiplatelet therapy and statin therapy. Eligible participants were enrolled in the final analysis if they met the following criteria. Informed consent was obtained from participants or their legal representatives. This study was approved by the Ethics Committee of Nanjing First Hospital, Haimen Hospital Affiliated to Nantong University as well as Nantong Third Peoples Hospital.

Inclusion criteria:

1. Admission within $4.5 \mathrm{~h}$ after onset;

2. Treated with intravenous thrombolysis;

3. Eighteen years or older.

Exclusion criteria:

1. Severe inflammatory diseases or infectious diseases; 
2. Incomplete clinical data;

3. The neurological deficits of patients cannot be evaluated over the following $24 \mathrm{~h}$ after admission.

\section{Data acquisition}

On the day of admission, all the participants underwent standard assessments of demographic characteristics (age, sex, and body mass index), vascular risk factors (hypertension, diabetes mellitus, dyslipidemia, atrial fibrillation, current smoking, current drinking, previous stroke, peripheral artery disease, and coronary artery disease), medication use history (previous antiplatelet, previous anticoagulation, and previous statin), clinical assessment (stroke severity, blood pressure, hemorrhagic transformation $[\mathrm{HT}]$, onset to treatment time [OTT], proximal arterial occlusion [PAO], and endovascular treatment), stroke subtype, lesion location, and laboratory data. Systolic blood pressure and diastolic blood pressure were measured and recorded immediately after admission. Computed tomography, magnetic resonance, electrocardiogram, echocardiography, carotid ultrasonography, and transcranial Doppler were performed for assessing the lesion location, stroke subtype, HT, and PAO. Stroke subtype was classified according to Trial of Org 10172 in Acute Stroke Treatment (TOAST) criteria [22]. Laboratory data included total cholesterol (TC), triglyceride (TG), high-density lipoprotein (HDL), lowdensity lipoprotein (LDL), fasting blood glucose (FBG), hypersensitive $\mathrm{C}$-reactive protein (Hs-CRP), NLR, PLR, and LMR.

\section{Measurement of composite inflammatory ratios from blood cell counts}

Blood cell counts, including total leukocyte counts, neutrophil counts, lymphocyte counts, monocyte counts, and platelet counts, were sampled from each participant in emergency room on admission before intravenous thrombolysis. Then, the cell counts were analyzed by an auto-analyzer (XE-2100, Sysmex, Kobe, Japan) and utilized to calculate composite inflammatory ratios (including NLR, PLR, and LMR). NLR was calculated as neutrophil counts/lymphocyte counts. PLR was calculated as platelet counts/lymphocyte counts. LMR was calculated as lymphocyte counts/monocyte counts.

\section{Definition of post-thrombolysis early neurological deterioration and early neurological improvement}

Stroke severity was assessed using NIHSS score on the day of admission and continued 2-3 times over the following $24 \mathrm{~h}$ after admission by two certified neurologists. All the certified neurologists in the three centers underwent unified training for NIHSS score evaluation and were blind to our study. In case of disagreement about the NIHSS score evaluation, a third neurologist in this center was invited for a final decision. Post-thrombolysis END was defined as an increase in the NIHSS score by $\geq 4$ points in the total score within $24 \mathrm{~h}$ after thrombolysis [7, 23, 24]. Meanwhile, post-thrombolysis ENI was defined as a decrease in the NIHSS score by $\geq 4$ points in the total score or a complete resolution of neurological deficits within $24 \mathrm{~h}$ after thrombolysis [25-27].

\section{Statistical analysis}

Statistical analyses were performed using $\mathrm{R}$ version 4.0.3 software (http://www.R-project.org/). All participants were categorized into 3 groups according to post-thrombolysis early neurological outcomes (END group, neither END nor ENI group as well as ENI group). Categorical variables were expressed as $n$ (\%), and continuous variables were expressed as means (standard deviation, SD) or medians (interquartile range, IQR). Differences in baseline characteristics between groups were analyzed using one-way ANOVA test or Mann-Whitney $U$ test for continuous variables as well as the chi-squared test or Fisher's exact test for categorical variables, as appropriate. We used the violin plots to show the distribution of NLR, PLR, and LMR among the END group, neither the END nor ENI group as well as the ENI group. We also used crude model analyses to detect the risk factors of END and ENI. By setting "neither END nor ENI" as the reference category, multinomial logistic regression analysis was adjusted for all potential confounders with statistically significant association at $P<0.05$ in comparison of baseline demographics and clinical characteristics among three groups according to early neurological outcomes. A MedCalc 15.6.0 (MedCalc Software Acacialaan 22, B-8400 Ostend, Belgium) packet program was used to obtain receiver operating characteristic (ROC) curve to test the overall discriminative ability of NLR, PLR, and LMR for post-thrombolysis END as well as post-thrombolysis ENI. Youden Index was calculated as the Sensitivity+Specificity-1. A two-tailed value of $P<0.05$ was considered significant.

\section{Results}

From April 2016 to September 2019, a total of 1235 AIS patients treated with intravenous thrombolysis were screened for $24 \mathrm{~h}$ in this study (Additional file 1: Figure S1). Ninety-two patients' neurological deficits could not be evaluated over the following $24 \mathrm{~h}$ after admission. Meanwhile, eighty-three patients were excluded for the following reasons: nineteen patients were excluded for severe inflammatory or infectious diseases, and sixtyfour patients were excluded for incomplete data. A total of 1060 subjects were included for the final analysis.

After admission, post-thrombolysis END was observed in 193 patients (18.2\%), and ENI was observed in 398 patients (37.5\%), respectively. Baseline characteristics of the study participants according to post-thrombolysis 
early neurological outcomes (END group, neither the END nor ENI group as well as the ENI group) are provided in Table 1. Significant differences among the three group are described as follows: age $(P=0.001)$, previous antiplatelet $(P=0.002)$, NIHSS $(P=0.001)$, diastolic blood pressure $(P=0.034)$, HT $(P=0.001)$, OTT $(P=$ $0.001)$, PAO $(P=0.001)$, stroke subtype $(P=0.001)$, FBG $(P=0.001)$, Hs-CRP $(P=0.001)$, NLR $(P=0.001)$, $\operatorname{PLR}(P=0.001)$, and LMR $(P=0.014)$. Figure 1 showed the violin plots of NLR, PLR, and LMR among three groups. The patients in the END group had elevated levels of NLR and PLR than those patients in the neither END nor ENI group. Compared to the patients in the ENI group, the patients in the END group possessed higher levels of NLR, PLR, and lower levels of LMR. What is more, the patients in the neither the END nor ENI group owned upgraded levels of NLR than the patients in the ENI group (all $P<0.05$ ).

Table 2 illustrated the results of crude models for post-thrombolysis END and post-thrombolysis ENI. Crude models for END showed that age, previous antiplatelet, NIHSS, HT, OTT, PAO, stroke subtype, FBG, Hs-CRP, NLR, PLR, and LMR might be associated with post-thrombolysis END $(P<0.05)$. Furthermore, crude models also exhibited that age, diabetes mellitus, previous antiplatelet, diastolic blood pressure, HT, OTT, PAO, NLR, PLR, and LMR might be related to postthrombolysis ENI $(P<0.05)$.

Table 3 displayed the results of the multiple logistic regression model for post-thrombolysis END and postthrombolysis ENI. After adjustment for all potential confounders, NLR (odds ratio [OR], 1.385; 95\% confidence interval $[\mathrm{CI}] 1.238-1.551, P=0.001$ ), PLR (OR, 1.013; 95\% CI 1.009-1.016, $P=0.001$ ), and LMR (OR, 0.680 ; $95 \%$ CI $0.560-0.825, P=0.001$ ) were identified as independent factors for post-thrombolysis END. What is more, NLR (OR, 0.713; 95\% CI 0.643-0.791, $P=$ 0.001) remained an independent factor for postthrombolysis ENI.

ROC curves, which were depicted in Fig. 2, were used to test the overall discriminative ability of these three composite inflammatory ratios for outcomes. We observed that the area under curve (AUC) of NLR, PLR, and LMR to discriminate post-thrombolysis END were 0.763 (95\% CI, 0.736-0.788), 0.703 (95\% CI, $0.675-$ 0.730), and 0.551 (95\% CI, 0.521-0.581) (Fig. 2a). To predict post-thrombolysis END, AUC of NLR was superior to PLR (0.763 versus $0.703, P=0.010)$ and LMR (0.763 versus $0.551, P=0.001)$. Moreover, AUC of PLR was superior to LMR (0.703 versus 0.551, $P=0.001$ ). Meanwhile, the AUC of NLR, PLR, and LMR to discriminate post-thrombolysis ENI were 0.695 (95\% CI, $0.666-$ 0.722 ), 0.530 (95\% CI, 0.499-0.560), and 0.547 (95\% CI, 0.516-0.577) (Fig. 2b). To predict post-thrombolysis
ENI, AUC of NLR was superior to PLR (0.695 versus $0.530, P=0.001)$ and $\operatorname{LMR}(0.695$ versus $0.547, P=$ $0.001)$. However, there was no significant difference between the AUC of PLR and LMR (0.530 versus 0.547, $P=0.461)$. We also established optimal cutoff values at which the Youden index was highest. The details of optimal cutoff values for NLR, PLR, and LMR as predictors of post-thrombolysis END and ENI were described in Additional file 2: Table S1.

\section{Discussion}

To our knowledge, this is the first study with relatively large samples to investigate the association between composite inflammatory ratios before thrombolysis and post-thrombolysis early neurological outcomes. Our study showed that the prevalence of post-thrombolysis END and post-thrombolysis ENI were $18.2 \%$ as well as $37.5 \%$, respectively. This prevalence is in line with the previous researches [24-28]. In this observational study, we found NLR, PLR, and LMR were associated with post-thrombolysis END. Moreover, NLR was related to post-thrombolysis ENI. In general, a biomarker with 0.7 $<$ AUC < 0.9 indicates a moderate diagnostic value. Therefore, NLR and PLR may be capable of predicting post-thrombolysis END.

During recent years, neuroinflammation has drawn more and more attention, and numerous studies have confirmed that inflammatory mechanisms play crucial roles in the pathogenesis and progression of ischemic stroke [29-34]. Peripheral leukocytes are guided by the inflammatory cytokines and chemokines, which are released from ischemic tissues [35]. Conversely, peripheral leukocytes may affect ischemic tissues as well [35]. Lymphocyte counts have been considered to have neuroprotective effect and contribute to neurological function improvement [35]. Both peripheral monocytes and neutrophils could perform as the source of matrix metalloproteinase-9, which would lead to HT and symptomatic deterioration [7, 36, 37]. Furthermore, it is reported that neutrophils can induce free oxygen radicals and cause brain injury [38]. Meanwhile, AIS may result in platelet function abnormality, and excessive activation and accumulation of platelets could hamper stroke recovery [39]. NLR, PLR, and LMR are three composite ratios in the combination of different inflammatory parameters, hence they may be able to provide more information about immunological activities during the pathogenesis of ischemic stroke. What is more, these three composite inflammatory ratios can be calculated from blood cell counts, so they are relatively accessible. Previous studies exhibited that NLR, PLR, and LMR could predict the clinical outcome in AIS patients [16, 18, 40]. NLR and LMR can also foretell HT after ischemic stroke [41, 42]. Moreover, high NLR and PLR might 
Table 1 Demographics and clinical characteristics of the subgroup according to early neurological outcomes

\begin{tabular}{|c|c|c|c|c|}
\hline Variable & $\begin{array}{l}\text { END group } \\
(n=193)\end{array}$ & $\begin{array}{l}\text { Neither END nor ENI } \\
\text { group }(n=469)\end{array}$ & $\begin{array}{l}\text { ENI group } \\
(n=398)\end{array}$ & $P$ \\
\hline \multicolumn{5}{|l|}{ Demographic characteristics } \\
\hline Age, years & $73.2 \pm 11.5$ & $69.6 \pm 12.0^{\mathrm{a}}$ & $68.1 \pm 12.1^{b}$ & 0.001 \\
\hline Male, $n(\%)$ & $121(62.7)$ & $319(68.0)$ & $260(65.3)$ & 0.303 \\
\hline $\mathrm{BMl}, \mathrm{kg} / \mathrm{m}^{2}$ & $24.4 \pm 3.6$ & $24.1 \pm 3.5$ & $23.9 \pm 3.6$ & 0.242 \\
\hline \multicolumn{5}{|l|}{ Vascular risk factors, $n$ (\%) } \\
\hline Hypertension & $132(68.4)$ & $315(67.2)$ & $275(69.1)$ & 0.816 \\
\hline Diabetes mellitus & $51(26.4)$ & $116(24.7)$ & $77(19.3)^{b}$ & 0.079 \\
\hline Dyslipidemia & $51(26.4)$ & $120(25.6)$ & $114(28.6)$ & 0.592 \\
\hline Atrial fibrillation & $49(25.4)$ & $96(20.5)$ & $75(18.8)$ & 0.180 \\
\hline Current smoking & $59(30.6)$ & $149(31.8)$ & $129(32.4)$ & 0.903 \\
\hline Current drinking & $69(35.8)$ & $145(30.9)$ & $129(32.4)$ & 0.482 \\
\hline Previous stroke & $38(19.7)$ & $114(24.3)$ & $80(20.1)$ & 0.238 \\
\hline Peripheral artery disease & $11(5.7)$ & $30(6.4)$ & $30(7.5)$ & 0.662 \\
\hline Coronary artery disease & $38(19.7)$ & $83(17.7)$ & $81(20.4)$ & 0.603 \\
\hline \multicolumn{5}{|l|}{ Medication use history, $n$ (\%) } \\
\hline Previous antiplatelet & $21(10.9)$ & $88(18.8)^{a}$ & $91(22.9)^{b}$ & 0.002 \\
\hline Previous statin & $15(7.8)$ & $49(10.4)$ & $42(10.6)$ & 0.521 \\
\hline Previous anticoagulation & $5(2.6)$ & $11(2.3)$ & $5(1.3)$ & 0.391 \\
\hline \multicolumn{5}{|l|}{ Clinical assessment } \\
\hline NIHSS, score & $11(5,17)$ & $5(3,12)^{a}$ & $8(4,14)^{b, c}$ & 0.001 \\
\hline $\mathrm{SBP}, \mathrm{mmHg}$ & $146.9 \pm 23.6$ & $151.8 \pm 25.7$ & $148.3 \pm 22.6$ & 0.171 \\
\hline $\mathrm{DBP}, \mathrm{mmHg}$ & $87.8 \pm 16.2$ & $89.4 \pm 14.4$ & $86.8 \pm 14.6^{c}$ & 0.034 \\
\hline $\mathrm{HT}, n(\%)$ & $32(16.6)$ & $37(7.9)^{a}$ & $13(3.3)^{b, c}$ & 0.001 \\
\hline OTT, minute & $165.00(120.00,200.00)$ & $160.00(115.00,200.00)$ & $130.00(90.00,180.00)^{b, c}$ & 0.001 \\
\hline $\mathrm{PAO}, n(\%)$ & 77 (39.9) & $134(28.6)^{a}$ & $91(22.9)^{b}$ & 0.001 \\
\hline Endovascular treatment, $n(\%)$ & $43(22.3)$ & $77(16.4)$ & $65(16.3)$ & 0.148 \\
\hline Stroke subtype, $n(\%)$ & & & & 0.001 \\
\hline LAA & $73(37.8)$ & $169(36.0)$ & $147(36.9)$ & \\
\hline CE & $62(32.1)$ & $120(25.6)$ & $132(33.2)$ & \\
\hline $\mathrm{SAO}$ & $27(14.0)$ & $138(29.4)^{a}$ & 83 (20.9) & \\
\hline SOE & $8(4.1)$ & $16(3.4)$ & $8(2.0)$ & \\
\hline SUE & $23(12.0)$ & $26(5.6)^{\mathrm{a}}$ & $28(7.0)$ & \\
\hline Lesion location, $n$ (\%) & & & & 0.616 \\
\hline Anterior circulation & $149(77.2)$ & $357(76.1)$ & $294(73.9)$ & \\
\hline Posterior circulation & $44(22.8)$ & $112(23.9)$ & $104(26.1)$ & \\
\hline \multicolumn{5}{|l|}{ Laboratory data } \\
\hline $\mathrm{TC}, \mathrm{mmol} / \mathrm{L}$ & $4.41 \pm 1.11$ & $4.38 \pm 1.11$ & $4.32 \pm 1.10$ & 0.591 \\
\hline $\mathrm{TG}, \mathrm{mmol} / \mathrm{L}$ & $1.14(0.82,1.68)$ & $1.19(0.86,1.70)$ & $1.20(0.85,1.75)$ & 0.744 \\
\hline $\mathrm{HDL}, \mathrm{mmol} / \mathrm{L}$ & $1.21 \pm 0.68$ & $1.14 \pm 0.36$ & $1.13 \pm 0.49$ & 0.214 \\
\hline $\mathrm{LDL}, \mathrm{mmol} / \mathrm{L}$ & $2.53(1.95,3.20)$ & $2.63(1.98,3.23)$ & $2.59(1.96,3.27)$ & 0.733 \\
\hline $\mathrm{FBG}, \mathrm{mmol} / \mathrm{L}$ & $7.30 \pm 2.95$ & $6.28 \pm 2.47^{\mathrm{a}}$ & $6.86 \pm 3.19^{c}$ & 0.001 \\
\hline $\mathrm{Hs}-\mathrm{CRP}, \mathrm{mg} / \mathrm{L}$ & $7.25(2.66,14.55)$ & $4.28(2.11,7.55)^{a}$ & $4.85(2.16,8.61)^{b}$ & 0.001 \\
\hline NLR & $6.09(4.43,8.02)$ & $4.15(3.14,5.26)^{\mathrm{a}}$ & $3.17(2.54,4.22)^{b, c}$ & 0.001 \\
\hline
\end{tabular}


Table 1 Demographics and clinical characteristics of the subgroup according to early neurological outcomes (Continued)

\begin{tabular}{|c|c|c|c|c|}
\hline Variable & $\begin{array}{l}\text { END group } \\
(n=193)\end{array}$ & $\begin{array}{l}\text { Neither END nor ENI } \\
\text { group }(n=469)\end{array}$ & $\begin{array}{l}\text { ENI group } \\
(n=398)\end{array}$ & $P$ \\
\hline PLR & $179.1(122.1,251.1)$ & $126.7(98.8,158.0)^{a}$ & $133.1(102.5,168.2)^{b}$ & 0.001 \\
\hline LMR & $3.03(2.50,4.13)$ & $3.36(2.48,4.34)$ & $3.63(2.61,4.52)^{b}$ & 0.014 \\
\hline
\end{tabular}

Abbreviations: END early neurological deterioration, ENI, early neurological improvement, $B M I$ body mass index, NIHSS National Institute of Health Stroke Scale, SBP systolic blood pressure, DBP diastolic blood pressure, $H T$ hemorrhagic transformation, $O T T$ onset to treatment time, $P A O$ proximal arterial occlusion, $L A A$ largeartery atherosclerosis, CE cardioembolism, SAO small-artery occlusion, SOE stroke of other determined etiology, SUE stroke of undetermined etiology, TC total cholesterol, TG triglyceride, $L D L$ low-density lipoprotein, $H D L$ high-density lipoprotein, FBG fasting blood glucose, $H s$-CRP hyper-sensitive C-reactive protein, NLR neutrophil-lymphocyte ratio, PLR platelet-lymphocyte ratio, LMR lymphocyte-monocyte ratio

${ }^{a}$ This variable is different significantly between END group and neither END nor ENI group, $P<0.05$

${ }^{b}$ This variable is different significantly between ENI group and END group, $P<0.05$

${ }^{C}$ This variable is different significantly between neither END nor ENI group and ENI group, $P<0.05$

be associated with symptomatic internal carotid artery stenosis [43]. Nam and colleagues found that upgraded NLR may portend stroke-associated pneumonia [44]. In addition, elevated levels of PLR are associated with poststroke depression according to the study of Huang et al. [45]. The findings of our study have supplemented the roles of NLR, PLR, and LMR in cerebrovascular disease and provide new ideas for clinical practice, too.

In this study, we also discovered that small artery occlusion subtype, stroke of undetermined etiology subtype (both take the large-artery atherosclerosis subtype as the reference category), baseline NIHSS, FBG, and Hs-CRP were related to post-thrombolysis END. In addition, multiple logistic regression models showed that age, diastolic blood pressure, HT, OTT, and PAO were connected with post-thrombolysis ENI. Previous researches confirmed that age, elevated levels of baseline NIHSS, OTT, and FBG may be able to be the risk factors of neurological recovery [7, 8, 46]. Moreover, HT could serve as one reason for END [6, 47], and PAO may lead to symptoms worsening and poor prognosis in AIS patients [48]. Hu et al. found that diastolic blood pressure might be associated with END [49]. One current study discovered that TOAST type of large-artery atherosclerosis was one of independent predictors for END [50], which hinted that the patients with other subtypes might be less likely to develop END than those patients with large-artery atherosclerosis subtype. However, the mechanisms of stroke subtype in the post-thrombolysis END still remain uncertain, and future studies are warranted to figure out the relationship between TOAST subtype and early neurological outcomes. Serum Hs-CRP could also function as an inflammatory biomarker. Our previous study showed that Hs-CRP could predict progressive motor deficits, a subtype of END, in patients with penetrating artery infarctions [51]. Moreover, there were

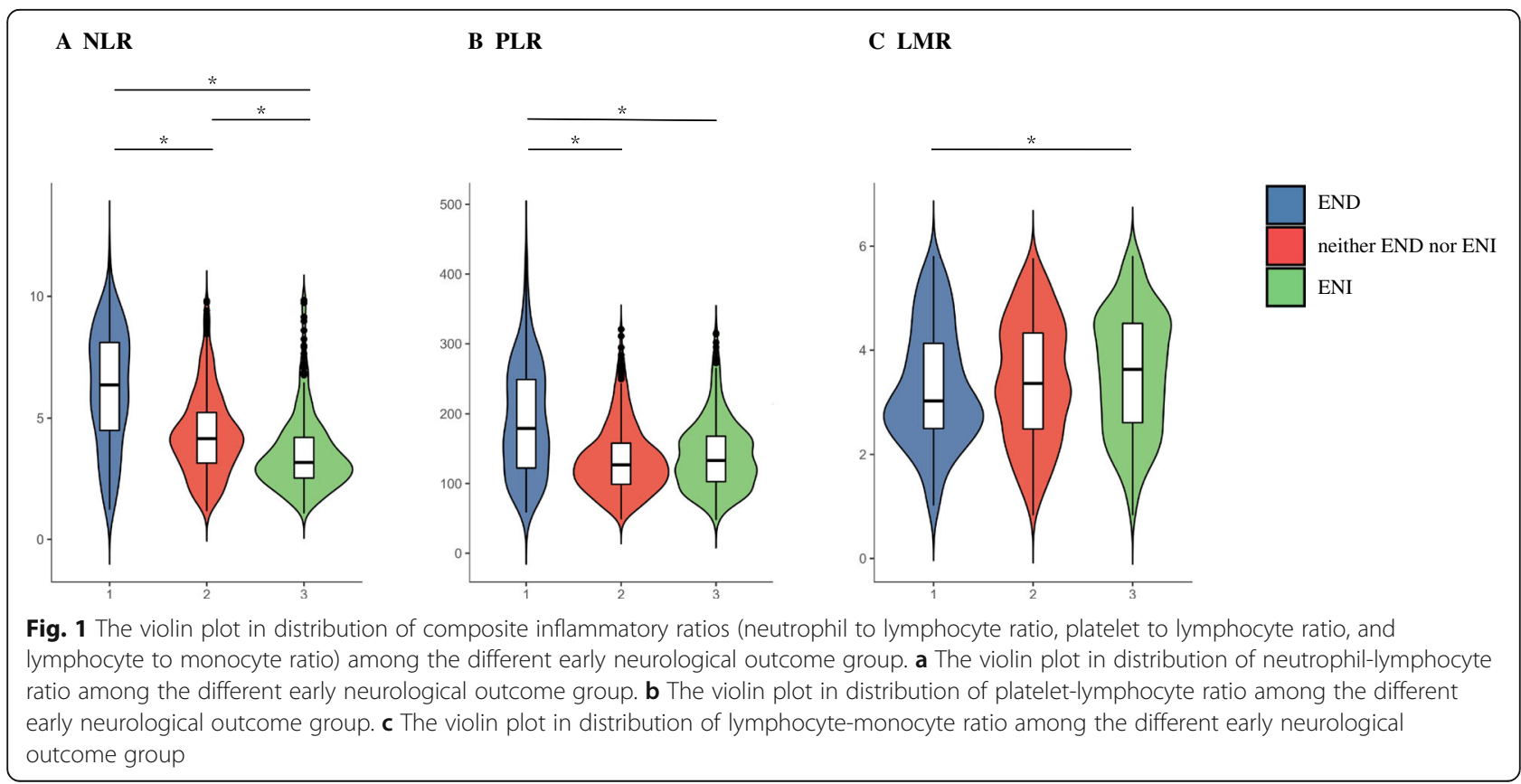


Table 2 Univariate Logistic regression analysis for risk factors with post-thrombolysis END and post-thrombolysis ENI

\begin{tabular}{|c|c|c|c|c|}
\hline \multirow[t]{2}{*}{ Variable } & \multicolumn{2}{|c|}{ Crude model for post-thrombolysis END } & \multicolumn{2}{|c|}{ Crude model for post-thrombolysis ENI } \\
\hline & OR $(95 \% \mathrm{Cl})$ & $P$ & OR $(95 \% \mathrm{Cl})$ & $P$ \\
\hline \multicolumn{5}{|l|}{ Demographic characteristics } \\
\hline Age & $1.033(1.018-1.048)$ & 0.001 & $0.982(0.972-0.993)$ & 0.002 \\
\hline Male & $0.813(0.587-1.125)$ & 0.211 & $0.950(0.729-1.237)$ & 0.702 \\
\hline $\mathrm{BMI}$ & $1.032(0.987-1.079)$ & 0.169 & $0.974(0.940-1.010)$ & 0.159 \\
\hline \multicolumn{5}{|l|}{ Vascular risk factors } \\
\hline Hypertension & $1.033(0.737-1.447)$ & 0.851 & $1.070(0.819-1.399)$ & 0.629 \\
\hline Diabetes mellitus & $1.254(0.877-1.794)$ & 0.215 & $0.711(0.524-0.964)$ & 0.028 \\
\hline Dyslipidemia & $0.972(0.682-1.383)$ & 0.873 & $1.153(0.872-1.523)$ & 0.317 \\
\hline Atrial fibrillation & $1.385(0.962-1.995)$ & 0.080 & $0.828(0.606-1.130)$ & 0.235 \\
\hline Current smoking & $0.933(0.665-1.308)$ & 0.687 & $1.047(0.802-1.366)$ & 0.737 \\
\hline Current drinking & $1.204(0.868-1.671)$ & 0.226 & $1.004(0.770-1.309)$ & 0.977 \\
\hline Previous stroke & $0.847(0.574-1.249)$ & 0.402 & $0.848(0.625-1.150)$ & 0.288 \\
\hline Peripheral artery disease & $0.813(0.419-1.577)$ & 0.540 & $1.235(0.758-2.012)$ & 0.397 \\
\hline Coronary artery disease & 1.049 (0.708-1.555) & 0.810 & $1.140(0.833-1.561)$ & 0.412 \\
\hline \multicolumn{5}{|l|}{ Medication use history } \\
\hline Previous antiplatelet & $0.469(0.290-0.760)$ & 0.002 & 1.504 (1.102-2.053) & 0.010 \\
\hline Previous anticoagulation & $1.415(0.512-3.909)$ & 0.504 & $0.514(0.187-1.413)$ & 0.197 \\
\hline Previous statin & $0.848(0.638-1.127)$ & 0.256 & $1.050(0.855-1.289)$ & 0.642 \\
\hline \multicolumn{5}{|l|}{ Clinical assessment } \\
\hline $\mathrm{NIHSS}$ & $1.051(1.030-1.072)$ & 0.001 & 1.007 (0.990-1.024) & 0.446 \\
\hline SBP & $0.995(0.989-1.002)$ & 0.195 & $0.998(0.993-1.002)$ & 0.359 \\
\hline DBP & $0.998(0.993-1.009)$ & 0.737 & $0.990(0.982-0.999)$ & 0.023 \\
\hline $\mathrm{HT}$ & $3.248(2.020-5.221)$ & 0.001 & $0.290(0.158-0.532)$ & 0.001 \\
\hline OTT & $1.004(1.001-1.006)$ & 0.011 & $0.993(0.990-0.995)$ & 0.001 \\
\hline $\mathrm{PAO}$ & $1.894(1.368-2.623)$ & 0.001 & $0.634(0.476-0.843)$ & 0.002 \\
\hline Endovascular treatment & $1.464(0.997-2.148)$ & 0.052 & $0.882(0.633-1.228)$ & 0.456 \\
\hline \multicolumn{5}{|l|}{ Stroke subtype } \\
\hline LAA & Reference & & Reference & \\
\hline CE & $1.065(0.731-1.552)$ & 0.743 & $1.194(0.881-1.618)$ & 0.252 \\
\hline $\mathrm{SAO}$ & $0.529(0.329-0.849)$ & 0.008 & $0.828(0.592-1.157)$ & 0.268 \\
\hline SOE & $1.443(0.623-3.341)$ & 0.392 & $0.549(0.240-1.253)$ & 0.154 \\
\hline SUE & $1.844(1.063-3.197)$ & 0.029 & $0.941(0.566-1.563)$ & 0.813 \\
\hline \multicolumn{5}{|l|}{ Lesion location } \\
\hline Anterior circulation & Reference & & Reference & \\
\hline Posterior circulation & $0.890(0.615-1.288)$ & 0.537 & $1.147(0.861-1.528)$ & 0.347 \\
\hline \multicolumn{5}{|l|}{ Laboratory data } \\
\hline $\mathrm{TC}$ & $1.003(0.996-1.010)$ & 0.447 & $0.999(0.994-1.003)$ & 0.591 \\
\hline TG & $1.000(0.998-1.002)$ & 0.623 & $1.001(0.996-1.005)$ & 0.655 \\
\hline $\mathrm{HDL}$ & $1.255(0.953-1.654)$ & 0.106 & $0.889(0.664-1.190)$ & 0.428 \\
\hline LDL & $0.999(0.992-1.005)$ & 0.708 & $1.002(0.998-1.005)$ & 0.415 \\
\hline FBG & $1.084(1.032-1.138)$ & 0.002 & $1.035(0.991-1.080)$ & 0.119 \\
\hline $\mathrm{Hs}-\mathrm{CRP}$ & $1.033(1.018-1.048)$ & 0.001 & $0.997(0.984-1.010)$ & 0.630 \\
\hline NLR & 1.661 (1.528-1.807) & 0.001 & $0.678(0.626-0.735)$ & 0.001 \\
\hline
\end{tabular}


Table 2 Univariate Logistic regression analysis for risk factors with post-thrombolysis END and post-thrombolysis ENI (Continued)

\begin{tabular}{|c|c|c|c|c|}
\hline \multirow[t]{2}{*}{ Variable } & \multicolumn{2}{|c|}{ Crude model for post-thrombolysis END } & \multicolumn{2}{|c|}{ Crude model for post-thrombolysis ENI } \\
\hline & OR $(95 \% \mathrm{Cl})$ & $P$ & OR $(95 \% \mathrm{Cl})$ & $P$ \\
\hline PLR & $1.014(1.011-1.017)$ & 0.001 & $0.997(0.995-0.999)$ & 0.004 \\
\hline LMR & $0.869(0.758-0.996)$ & 0.043 & $1.152(1.033-1.284)$ & 0.011 \\
\hline
\end{tabular}

Abbreviation: END early neurological deterioration, ENI early neurological improvement, OR odds ratio, $C l$ confidence interval, $B M I$ body mass index, $N I H S S$ National Institute of Health Stroke Scale, SBP systolic blood pressure, DBP diastolic blood pressure, $H T$ hemorrhagic transformation, $O T T$ onset to treatment time, $P A O$ proximal arterial occlusion, $L A A$ large-artery atherosclerosis, CE cardioembolism, SAO small-artery occlusion, SOE stroke of other determined etiology, SUE, stroke of undetermined etiology, $T C$ total cholesterol, $T G$ triglyceride, $L D L$ low-density lipoprotein, $H D L$ high-density lipoprotein, $F B G$ fasting blood glucose, $H s$ - $C R P$ hypersensitive C-reactive protein, NLR neutrophil-lymphocyte ratio, PLR platelet-lymphocyte ratio, $L M R$ lymphocyte-monocyte ratio

other studies that found serum Hs-CRP levels might be independently relevant with END, after adjustment for confounders $[7,52]$.

Our study has several potential limitations. Firstly, all the participants enrolled were Chinese patients treated with intravenous thrombolysis, so the associations between composite inflammatory ratios before thrombolysis and post-thrombolysis early neurological outcomes need to be tested in non-Chinese populations. Second, some risk factors that may be linked to END or ENI, such as serum homocysteine [53] and trimethylamine Noxide levels [54], were unavailable in this study. These biomarkers might serve as potential independent predictors for END. Therefore, we attempt to collect these variables prospectively. Third, it is reported that composite inflammatory ratios might be able to vary significantly during hospitalization. Our future research needs dynamic examination of composite inflammatory ratios, not only composite inflammatory ratios before thrombolysis. Moreover, there were more than one neurologist that assessed stroke severity, which would lead to bias on evaluation of NIHSS, even though these neurologists have undergone standardized training. In addition, machine learning, which could use algorithms based on statistical assumptions and mathematical rules to learn patterns [55], is a novel approach to make classifications for the most probable reason. We aim to apply machine learning approach to predict the neurological outcomes

Table 3 Multinomial logistic regression models for post-thrombolysis END and post-thrombolysis ENI

\begin{tabular}{|c|c|c|c|c|}
\hline \multirow[t]{2}{*}{ Variable } & \multicolumn{2}{|c|}{ Post-thrombolysis END } & \multicolumn{2}{|c|}{ Post-thrombolysis ENI } \\
\hline & OR $(95 \% \mathrm{Cl})$ & $P$ & OR $(95 \% \mathrm{Cl})$ & $P$ \\
\hline Age & $1.015(0.997-1.034)$ & 0.109 & $0.980(0.967-0.992)$ & 0.002 \\
\hline Previous antiplatelet & $0.654(0.363-1.179)$ & 0.158 & $1.249(0.865-1.804)$ & 0.235 \\
\hline NIHSS & $1.041(1.012-1.070)$ & 0.005 & 1.029 (0.997-1.055) & 0.102 \\
\hline DBP & $0.997(0.984-1.011)$ & 0.704 & 0.985 (0.974-0.995) & 0.003 \\
\hline HT & 1.637 (0.849-3.157) & 0.142 & $0.456(0.229-0.908)$ & 0.025 \\
\hline OTा & 1.001 (0.998-1.005) & 0.504 & 0.992 (0.990-0.995) & 0.001 \\
\hline PAO & $1.277(0.834-1.954)$ & 0.260 & $0.688(0.489-0.968)$ & 0.032 \\
\hline \multicolumn{5}{|l|}{ Stroke subtype } \\
\hline LAA & Reference & & Reference & \\
\hline CE & $0.988(0.287-3.402)$ & 0.984 & $0.365(0.123-1.080)$ & 0.069 \\
\hline SAO & $0.263(0.114-0.608)$ & 0.002 & $0.649(0.332-1.269)$ & 0.206 \\
\hline SOE & $0.477(0.222-1.023)$ & 0.057 & $1.229(0.640-2.360)$ & 0.535 \\
\hline SUE & $0.410(0.193-0.872)$ & 0.021 & $0.862(0.454-1.638)$ & 0.651 \\
\hline FBG & $1.112(1.038-1.191)$ & 0.003 & $1.054(0.996-1.116)$ & 0.070 \\
\hline $\mathrm{Hs}-\mathrm{CRP}$ & $1.036(1.015-1.057)$ & 0.002 & $1.013(0.995-1.032)$ & 0.156 \\
\hline NLR & $1.385(1.238-1.551)$ & 0.001 & $0.713(0.643-0.791)$ & 0.001 \\
\hline PLR & $1.013(1.009-1.016)$ & 0.001 & 1.005 (0.999-1.011) & 0.084 \\
\hline LMR & $0.680(0.560-0.825)$ & 0.001 & 1.023 (0.889-1.172) & 0.751 \\
\hline
\end{tabular}

Abbreviations: END early neurological deterioration, ENI early neurological improvement, OR odds ratio, CI confidence interval, NIHSS National Institute of Health Stroke Scale, DBP diastolic blood pressure, $H T$ hemorrhagic transformation, OTT onset to treatment time, PAO proximal arterial occlusion, LAA large-artery atherosclerosis, CE cardioembolism, SAO small-artery occlusion, SOE stroke of other determined etiology, SUE stroke of undetermined etiology, FBG fasting blood glucose, $H s$-CRP hyper-sensitive C-reactive protein, NLR neutrophil-lymphocyte ratio, PLR platelet-lymphocyte ratio, LMR lymphocyte-monocyte ratio 

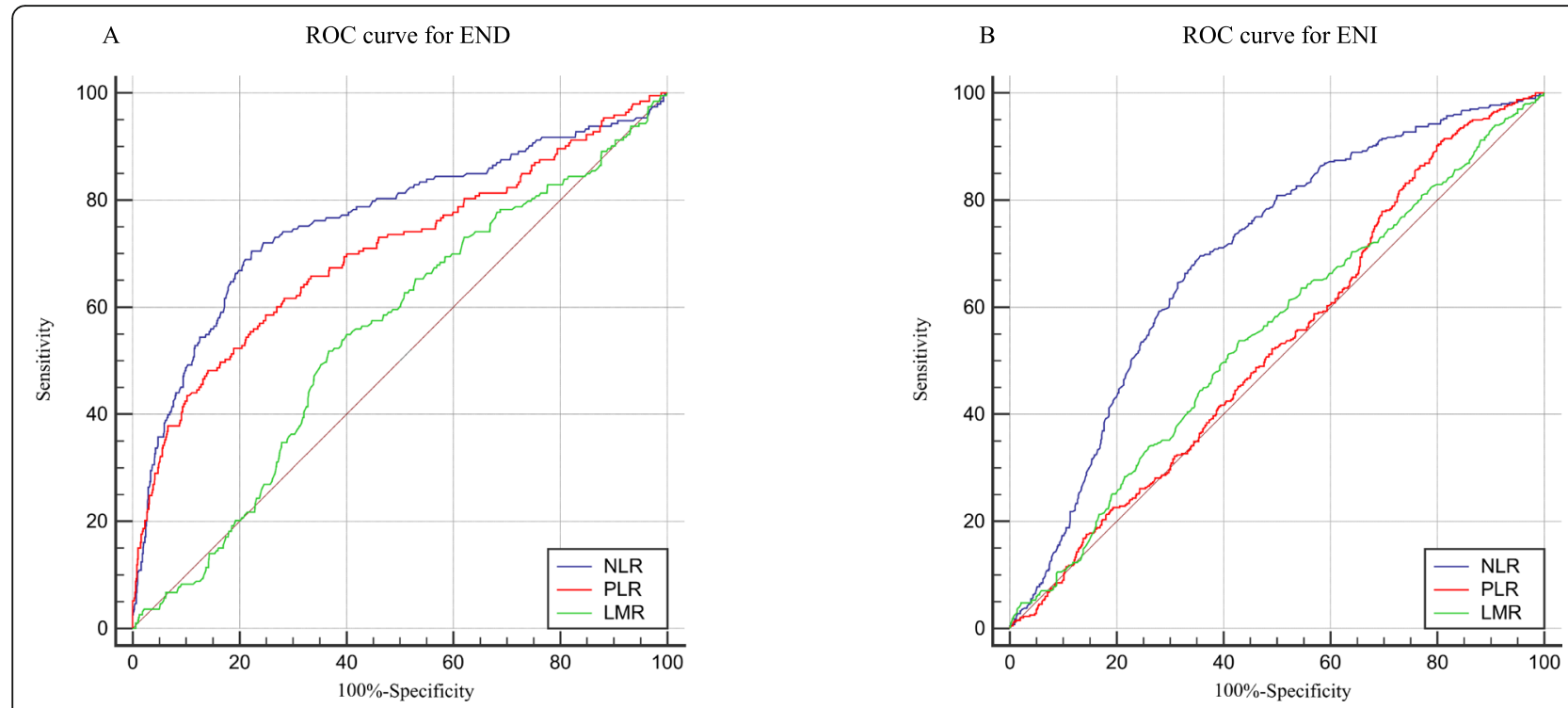

Fig. 2 Receiver operating characteristic curve for composite inflammatory ratios (neutrophil to lymphocyte ratio, platelet to lymphocyte ratio, and lymphocyte to monocyte ratio) to predict post-thrombolysis early neurological outcome. a Receiver operating characteristic curve to predict postthrombolysis early neurological deterioration. b Receiver operating characteristic curve to predict post-thrombolysis early neurological improvement

in the future study. Finally, we have not developed an independent validation cohort, which is able to provide more credibility. Therefore, it is meaningful and advantageous for us to establish an independent validation cohort in the coming research. Despite these limitations mentioned above, it is the first time to explore the relationship between composite inflammatory ratios before thrombolysis and post-thrombolysis early neurological outcomes, including END and ENI, with relatively large samples.

\section{Conclusion}

In summary, our study showed that NLR before thrombolysis, LMR before thrombolysis and PLR before thrombolysis were associated with post-thrombolysis END. Meanwhile, NLR before thrombolysis was related to post-thrombolysis ENI. What is more, NLR and PLR may have the ability to predict post-thrombolysis END. NLR, PLR, and LMR, which are easily available, might have utility as an inclusion criterion for future clinical trials about thrombolysis. Further investigations will be required to verify these results about post-thrombolysis early neurological outcomes.

\section{Supplementary Information}

The online version contains supplementary material available at https://doi. org/10.1186/s12974-021-02090-6.

Additional file 1: Figure S1. The flowchart of participants selection. Additional file 2: Table S1. The ROC curves for post-thrombolysis END and post-thrombolysis ENI.

Additional file 3: Table S2. The ROC curves for post-thrombolysis END.
Additional file 4: Table S3. The ROC curves for post-thrombolysis ENI.

\section{Abbreviations}

AIS: Acute ischemic stroke; ENI: Early neurological improvement; END: Early neurological deterioration; NLR: Neutrophil to lymphocyte ratio; PLR: Platelet to lymphocyte ratio; LMR: Lymphocyte to monocyte ratio; HT: Hemorrhagic transformation; OTT: Onset to treatment time; PAO: Proximal arterial occlusion; TOAST: Trial of Org 10172 in Acute Stroke Treatment; TC: Total cholesterol; TG: Triglyceride; HDL: High-density lipoprotein; LDL: Low-density lipoprotein; FBG: Fasting blood glucose; Hs-CRP: Hypersensitive C-reactive protein; OR: Odds ratio; 95\% Cl: 95\% Confidence interval; AUC: Area under the curve

\section{Acknowledgements}

We express our gratitude to all the researchers and patients who participated in this study.

\section{Authors' contributions}

Pengyu Gong was mainly involved in study design, data analysis, data interpretation, and manuscript preparation. Yukai Liu was mainly involved in data acquisition and data analysis. Yachi Gong, Gang Chen, Feng Zhou, Siyu Wang, Rui Duan, Wenxiu Chen, Meng Wang, Ting Huang, and Hongchao Shi were mainly involved in data acquisition. Xiaohao Zhang and Qiwen Deng were mainly involved in data analysis and manuscript preparation. Junshan Zhou, Teng Jiang, and Yingdong Zhang were mainly involved in study design, data interpretation, and manuscript preparation. The authors read and approved the final manuscript.

\section{Funding}

This study was supported by the Program of Nanjing Medical Science and Technology Development Foundation (ZDX16002, ZKX16050, YKK18097) and Medical Innovation Team of Jiangsu Province (CXTDA2017030).

\section{Availability of data and materials}

The data that support the findings of this study are available from the corresponding author upon reasonable request.

\section{Ethics approval and consent to participate}

This study was approved by the Ethics Committee of Nanjing First Hospital, Haimen Hospital Affiliated to Nantong University, and Nantong Third Peoples 
Hospital. Informed consent was obtained from participants or their legal representatives.

\section{Consent for publication}

All the authors agree to publish.

\section{Competing interests}

The authors declare that they have no competing interests.

\section{Author details}

${ }^{1}$ Department of Neurology, Nanjing First Hospital, Nanjing Medical University, Nanjing 210006, Jiangsu, China. ${ }^{2}$ Department of Gerontology, Nantong Third People's Hospital, Nantong University, Nantong 226006, Jiangsu, China. ${ }^{3}$ Department of Neurology, Haimen Hospital Affiliated to Nantong University, Nantong 226000, Jiangsu, China. ${ }^{4}$ Department of Neurology, Jinling Hospital, Medical School of Nanjing University, Nanjing 210000, Jiangsu, China. ${ }^{5}$ School of Basic Medicine and Clinical Pharmacy, China Pharmaceutical University, Nanjing 210000, Jiangsu, China. ${ }^{6}$ Department of Critical Care Medicine, Nanjing First Hospital, Nanjing Medical University, Nanjing 210006, Jiangsu, China.

Received: 25 November 2020 Accepted: 19 January 2021

\section{Published online: 20 February 2021}

\section{References}

1. Zhou M, Wang H, Zeng X, Yin P, Zhu J, Chen W, et al. Mortality, morbidity, and risk factors in China and its provinces, 1990-2017: a systematic analysis for the Global Burden of Disease Study 2017. Lancet. 2019;394:1145-58.

2. Wang $W$, Jiang $B$, Sun $H$, Ru $X$, Sun $D$, Wang $L$, et al. Prevalence, incidence, and mortality of stroke in China: results from a nationwide populationbased survey of 480687 adults. Circulation. 2017;135:759-71.

3. Hacke W, Kaste M, Bluhmki E, et al. Thrombolysis with alteplase 3 to 4.5 hours after acute ischemic stroke. N Engl J Med. 2008;359:1317-29.

4. The National Institute of Neurological Disorders and Stroke rt-PA Stroke Study Group. Tissue plasminogen activator for acute ischemic stroke. N Engl J Med. 1995;333:1581-7.

5. Powers WJ, Rabinstein AA, Ackerson T, et al. 2018 Guidelines for the early management of patients with acute ischemic stroke: a guideline for healthcare professionals from the American Heart Association/American Stroke Association. Stroke. 2018;49(3):e46-e110.

6. Seners P, Turc G, Oppenheim C, Baron JC. Incidence, causes and predictors of neurological deterioration occurring within $24 \mathrm{~h}$ following acute ischaemic stroke: a systematic review with pathophysiological implications. J Neurol Neurosurg Psychiatry. 2015;86(1):87-94.

7. Gong $P, X i e ~ Y$, Jiang $T$, et al. Neutrophil-lymphocyte ratio predicts postthrombolysis early neurological deterioration in acute ischemic stroke patients. Brain Behav. 2019;9(10):e01426.

8. Zhang X, Gong P, Sheng L, et al. Prognostic value of subclinical thyroid dysfunction in ischemic stroke patients treated with intravenous thrombolysis. Aging (Albany NY). 2019;11(17):6839-50.

9. Yeo LL, Paliwal P, Teoh HL, et al. Early and continuous neurologic improvements after intravenous thrombolysis are strong predictors of favorable long-term outcomes in acute ischemic stroke. J Stroke Cerebrovasc Dis. 2013;22(8):e590-6.

10. Mori M, Naganuma M, Okada Y, et al. Early neurological deterioration within 24 hours after intravenous rt-PA therapy for stroke patients: the Stroke Acute Management with Urgent Risk Factor Assessment and Improvement rt-PA Registry. Cerebrovasc Dis. 2012;34(2):140-6.

11. Schuhmann MK, Stoll G, Bieber M, et al. CD84 links T cell and platelet activity in cerebral thrombo-inflammation in acute stroke. Circ Res. 2020; 127(8):1023-35.

12. Parikh NS, Merkler AE, ladecola C. Inflammation, autoimmunity, infection, and stroke: epidemiology and lessons from therapeutic intervention. Stroke. 2020;51(3):711-8.

13. Stoll G, Nieswandt B. Thrombo-inflammation in acute ischaemic stroke implications for treatment. Nat Rev Neurol. 2019;15(8):473-81.

14. Dong X, Gao J, Zhang CY, Hayworth C, Frank M, Wang Z. Neutrophil membrane-derived nanovesicles alleviate inflammation to protect mouse brain injury from ischemic stroke. ACS Nano. 2019;13(2):1272-83.

15. Zhu B, Pan $Y$, Jing J, et al. Neutrophil counts, neutrophil ratio, and new stroke in minor ischemic stroke or TIA. Neurology. 2018;90(21):e1870-8.
16. Lux D, Alakbarzade V, Bridge $L$, et al. The association of neutrophillymphocyte ratio and lymphocyte-monocyte ratio with 3-month clinical outcome after mechanical thrombectomy following stroke. J Neuroinflammation. 2020;17(1):60.

17. Liu YL, Wu ZQ, Qu JF, et al. High neutrophil-to-lymphocyte ratio is a predictor of poor short-term outcome in patients with mild acute ischemic stroke receiving intravenous thrombolysis. Brain Behav. 2020;10(12):e01857.

18. $\mathrm{Xu} \mathrm{JH}, \mathrm{He} X \mathrm{X}, \mathrm{Li}$ Q, et al. Higher platelet-to-lymphocyte ratio is associated with worse outcomes after intravenous thrombolysis in acute ischaemic stroke. Front Neurol. 2019;10:1192.

19. Ren H, Han L, Liu H, Wang L, Liu X, Gao Y. Decreased lymphocyte-tomonocyte ratio predicts poor prognosis of acute ischemic stroke treated with thrombolysis. Med Sci Monit. 2017;23:5826-33.

20. Goyal N, Tsivgoulis G, Chang JJ, et al. Admission neutrophil-to-lymphocyte ratio as a prognostic biomarker of outcomes in large vessel occlusion strokes. Stroke. 2018;49(8):1985-7.

21. Curbelo J, Luquero Bueno S, Galván-Román JM, et al. Inflammation biomarkers in blood as mortality predictors in community-acquired pneumonia admitted patients: importance of comparison with neutrophil count percentage or neutrophil-lymphocyte ratio. Plos One. 2017;12(3): e0173947.

22. Adams HP Jr, Bendixen BH, Kappelle LJ, et al. Classification of subtype of acute ischemic stroke. Definitions for use in a multicenter clinical trial. TOAST. Trial of Org 10172 in Acute Stroke Treatment. Stroke. 1993; 24(1):35-41.

23. Yu WM, Abdul-Rahim AH, Cameron AC, et al. The incidence and associated factors of early neurological deterioration after thrombolysis: results from SITS registry. Stroke. 2020;51(9):2705-14.

24. Boulenoir N, Turc G, Henon $\mathrm{H}$, et al. Early neurological deterioration following thrombolysis for minor stroke with isolated internal carotid artery occlusion. Eur J Neurol. 2021;28(2):479-90

25. Logallo N, Novotny $\vee$, Assmus J, et al. Tenecteplase versus alteplase for management of acute ischaemic stroke (NOR-TEST): a phase 3, randomised, open-label, blinded endpoint trial. Lancet Neurol. 2017; 16(10):781-8.

26. Lin $\mathrm{CM}, \mathrm{Wu} \mathrm{HC}$, Wu YM, et al. Computed tomography angiography in acute stroke patients receiving recombinant tissue plasminogen activator: outcome and safety evaluations in an asian population. Cerebrovasc Dis. 2020;49(1):62-9.

27. Tian C, Ji Z, Xiang W, et al. Association of lower leukocyte count before thrombolysis with early neurological improvement in acute ischemic stroke patients. J Clin Neurosci. 2018;56:44-9.

28. Ajili N, Decroix JP, Preda C, et al. Impact of thrombolysis in acute ischaemic stroke without occlusion: an observational comparative study. Eur J Neurol. 2016:23(8):1380-6.

29. Chamorro Á, Dirnagl U, Urra X, Planas AM. Neuroprotection in acute stroke: targeting excitotoxicity, oxidative and nitrosative stress, and inflammation. Lancet Neurol. 2016;15(8):869-81.

30. Becker KJ, Buckwalter M. Stroke, inflammation and the immune response: dawn of a new era. Neurotherapeutics. 2016;13(4):659-60.

31. Shi K, Tian DC, Li ZG, Ducruet AF, Lawton MT, Shi FD. Global brain inflammation in stroke. Lancet Neurol. 2019;18(11):1058-66.

32. Anrather J, ladecola C. Inflammation and stroke: an overview. Neurotherapeutics. 2016;13(4):661-70

33. Shichita $\mathrm{T}$, Ito M, Morita $\mathrm{R}$, et al. MAFB prevents excess inflammation after ischemic stroke by accelerating clearance of damage signals through MSR1. Nat Med. 2017;23(6):723-32.

34. Rust R, Grönnert L, Schwab ME. Inflammation after stroke: a local rather than systemic response? Trends Neurosci. 2018;41(12):877-9.

35. Macrez R, Ali C, Toutirais $O$, et al. Stroke and the immune system: from pathophysiology to new therapeutic strategies. Lancet Neurol. 2011;10(5):471-80.

36. Duan Z, Wang H, Wang Z, et al. Neutrophil-lymphocyte ratio predicts functional and safety outcomes after endovascular treatment for acute ischemic stroke. Cerebrovasc Dis. 2018;45(5-6):221-7.

37. Yamamoto $Y$, Osanai T, Nishizaki F, et al. Matrix metalloprotein- 9 activation under cell-to-cell interaction between endothelial cells and monocytes: possible role of hypoxia and tumor necrosis factor-a. Heart Vessels. 2012; 27(6):624-33.

38. Ceulemans AG, Zgavc T, Kooijman R, Hachimi-Idrissi S, Sarre S, Michotte Y. The dual role of the neuroinflammatory response after ischemic stroke: modulatory effects of hypothermia. J Neuroinflammation. 2010;7:74. 
39. Xu XR, Zhang D, Oswald BE, et al. Platelets are versatile cells: new discoveries in hemostasis, thrombosis, immune responses, tumor metastasis and beyond. Crit Rev Clin Lab Sci. 2016;53(6):409-30.

40. Ren H, Liu X, Wang L, Gao Y. Lymphocyte-to-monocyte ratio: a novel predictor of the prognosis of acute ischemic stroke. J Stroke Cerebrovasc Dis. 2017:26(11):2595-602.

41. Zhang WB, Zeng YY, Wang F, Cheng L, Tang WJ, Wang XQ. A high neutrophil-to-lymphocyte ratio predicts hemorrhagic transformation of large atherosclerotic infarction in patients with acute ischemic stroke. Aging (Albany NY). 2020;12(3):2428-39.

42. Song $\mathrm{Q}$, Pan $\mathrm{R}$, Jin $\mathrm{Y}$, et al. Lymphocyte-to-monocyte ratio and risk of hemorrhagic transformation in patients with acute ischemic stroke. Neurol Sci. 2020;41(9):2511-20.

43. Massiot N, Lareyre F, Voury-Pons A, et al. High neutrophil to lymphocyte ratio and platelet to lymphocyte ratio are associated with symptomatic internal carotid artery stenosis. J Stroke Cerebrovasc Dis. 2019;28(1):76-83.

44. Nam KW, Kim TJ, Lee JS, et al. High neutrophil-to-lymphocyte ratio predicts stroke-associated pneumonia. Stroke. 2018;49(8):1886-92.

45. Huang $\mathrm{G}$, Chen $\mathrm{H}$, Wang $\mathrm{Q}$, et al. High platelet-to-lymphocyte ratio are associated with post-stroke depression. J Affect Disord. 2019;246:105-11.

46. Gong P, Zhang X, Gong Y, et al. A novel nomogram to predict early neurological deterioration in patients with acute ischaemic stroke. Eur J Neurol. 2020;27(10):1996-2005.

47. Siegler JE, Martin-Schild S. Early neurological deterioration (END) after stroke: the END depends on the definition. Int J Stroke. 2011;6(3):211-2.

48. Kim JT, Park MS, Chang J, Lee JS, Choi KH, Cho KH. Proximal arterial occlusion in acute ischemic stroke with low NIHSS scores should not be considered as mild stroke. Plos One. 2013;8(8):e70996.

49. Hu W, Liu D, Li Q, Wang L, Tang Q, Wang G. Decreasing serum 25hydroxyvitamin $\mathrm{D}$ levels and risk of early neurological deterioration in patients with ischemic stroke. Brain Behav. 2019;9(3):e01227.

50. Xu Y, Chen Y, Chen R, Zhao F, Wang P, Yu S. External validation of the WORSEN score for prediction the deterioration of acute ischemic stroke in a Chinese population. Front Neurol. 2020;11:482.

51. Gong P, Liu Y, Huang T, et al. The association between high-sensitivity Creactive protein at admission and progressive motor deficits in patients with penetrating artery infarctions. BMC Neurol. 2019;19(1):346.

52. Zhang $X$, Sun Z, Ding C, Tang Y, Jiang X, Xie Y, et al. Metabolic syndrome augments the risk of early neurological deterioration in acute ischemic stroke patients independent of inflammatory mediators: a hospital-based prospective study. Oxid Med Cell Longev. 2016;2016:8346301.

53. Kwon HM, Lee YS, Bae HJ, Kang DW. Homocysteine as a predictor of early neurological deterioration in acute ischemic stroke. Stroke. 2014r;45(3):871-3.

54. Hou L, Zhang Y, Zheng D, et al. Increasing trimethylamine N-oxide levels as a predictor of early neurological deterioration in patients with acute ischemic stroke. Neurol Res. 2020;42(2):153-8.

55. Morang'a CM, Amenga-Etego L, Bah SY, et al. Machine learning approaches classify clinical malaria outcomes based on haematological parameters. BMC Med. 2020;18(1):375.

\section{Publisher's Note}

Springer Nature remains neutral with regard to jurisdictional claims in published maps and institutional affiliations.

Ready to submit your research? Choose BMC and benefit from:

- fast, convenient online submission

- thorough peer review by experienced researchers in your field

- rapid publication on acceptance

- support for research data, including large and complex data types

- gold Open Access which fosters wider collaboration and increased citations

- maximum visibility for your research: over $100 \mathrm{M}$ website views per year

At BMC, research is always in progress.

Learn more biomedcentral.com/submissions 